\title{
Extended operation for recurrent thymic carcinoma presenting with intracaval growth and intracardiac extension
}

\author{
Tiziano De Giacomo, MD, Giuseppe Mazzesi, MD, Federico Venuta, MD, and G. F. Coloni, MD, Rome, Italy
}

T

hymic carcinoma is a relatively uncommon tumor that represents less than $1 \%$ of thymic malignancies. The prognosis is often poor, with a 5-year survival for all patients of between $31 \%$ and $50 \% .^{1}$ At present, a multimodality approach including aggressive surgical resection, platinumbased combination chemotherapy, and radiotherapy seems the preferred therapeutic strategy. Overall recurrence rate is very high and vascular invasion is particularly associated with poor prognosis. ${ }^{2,3}$ Only aggressive and complete resection yields long-term survival. We report the management of recurrent thymic carcinoma with extension into the right atrium, resulting from the progression of intracaval growth, 4 years after induction chemotherapy and successful total resection followed by chemoradiation treatment.

\section{Clinical Summary}

A 46-year-old man with sudden engorgement of neck veins, swelling of the face, and mild ocular myasthenia was admitted to our institution. Recurrence of thymic carcinoma was suspected inasmuch as the patient had undergone radical resection, with negative resection margins, of thymic carcinoma 4 years earlier, after induction chemotherapy. The patient received postoperative adjuvant therapy, including 6 cycles of platinum-based chemotherapy followed by $40 \mathrm{~Gy}$ of mediastinal irradiation. Contrast-enhanced computed tomographic scanning of the chest revealed an inhomogeneous mass almost completely obstructing the superior vena cava (SVC) and extending into the right atrium without evidence of tumor recurrence around the vessel, into the mediastinum, or in other sites. A magnetic resonance image confirmed the presence of a continuous pathologic tissue growing into the SVC and into the right atrium (Figure 1). A transthoracic echocardiogram showed a mass that almost completely filled the right atrium but was unable to show its relation to the great veins. A transesophageal echocardiogram confirmed the mass in the right atrium, which was connected to the mass into the SVC; no other significant abnormalities were found in the remaining cardiac chambers, valves, and great vessels.

The patient was operated on through a median resternotomy. Exploration of the mediastinum did not demonstrate extravascular

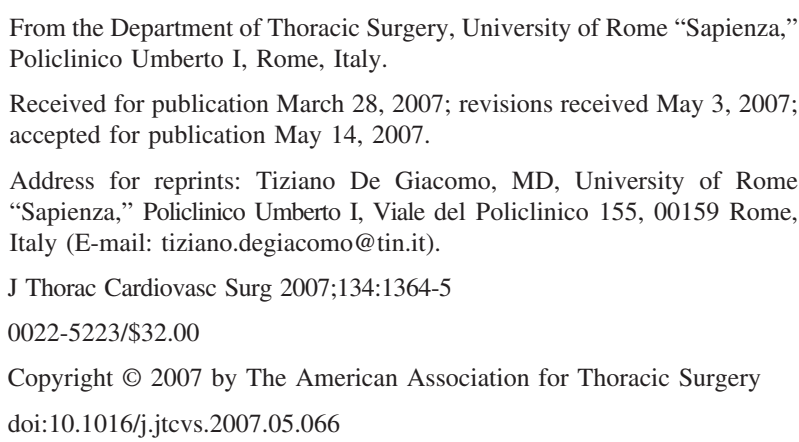

recurrence of the tumor or malignant lymphoadenopathies. Extracorporeal circulation was instituted by cannulating separately inferior vena cava and SVC immediately after the confluence of the innominate vessel over the edge of the intracaval mass. Opening the right atrium, without cardiac arrest, revealed a large tumor strictly adherent to the cava-atrial junction, completely obstructing the SVC. The mass was resected en bloc with the infiltrated portion of the atrial wall and the SVC.

The right atrium was reconstructed with a patch of bovine pericardium, and the same material was shaped into a tube, with a linear stapler, and used for SVC substitution (Figure 2). The postoperative course was uneventful. Anticoagulation therapy was started to prevent thrombosis of the extensive pericardial reconstruction of the SVC and right atrium.

Definitive pathologic examination showed that resection margins were negative and the tumor was a well-differentiated thymic carcinoma (B3 of the World Health Organization classification). A postoperative echocardiogram demonstrated that the right atrium was completely free of tumor, and a contrast-enhanced computed tomographic scan of the chest confirmed the normal flow in the SVC district.

Subsequent treatment included 4 cycles of platinum-based chemotherapy. One year after the operation the patient is alive and well without evidence of disease.

\section{Discussion}

The optimum treatment protocol for thymic carcinoma remains undetermined, although at present a multimodality approach involving aggressive surgical resection, platinum-based combination chemotherapy, and radiotherapy represents the preferred therapeutic strategy. Overall, prognosis is poor and reported recurrence rate very high $(65 \%$ at 5 years and $75 \%$ at 10 years $){ }^{2}$ Five-year

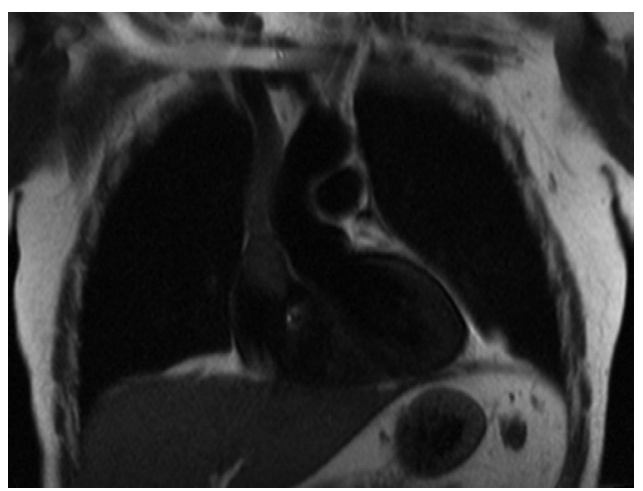

Figure 1. Magnetic resonance image of the chest showing the tumor growing into the superior vena cava and extending into the right atrium. 


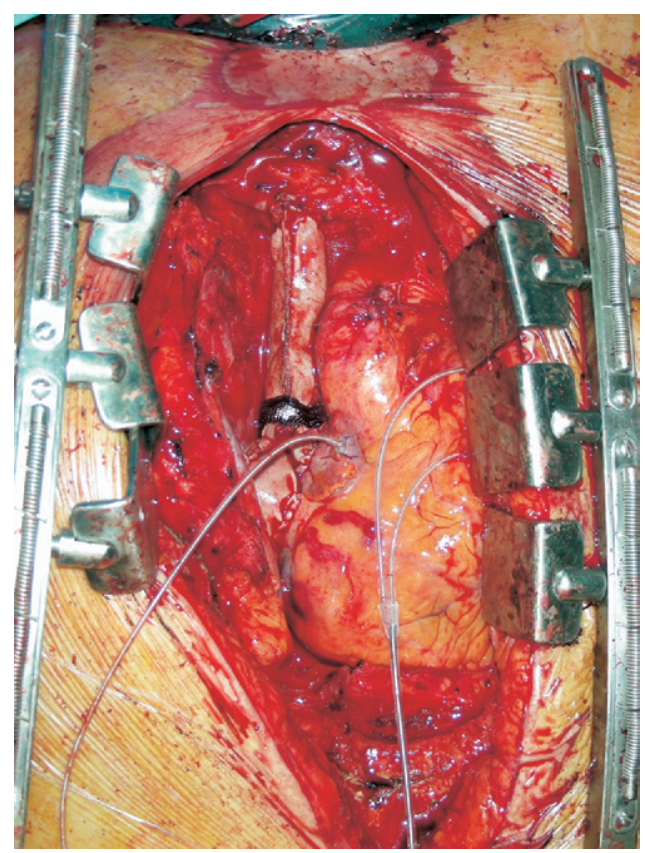

Figure 2. Intraoperative photograph at the end of the operation: the complete reconstruction of the superior vena cava with the pericardial tube and the patch on the right atrium are clearly visible.

survivals for total resection, subtotal resection, and inoperable groups are $67 \%, 30 \%$, and $24 \%$, respectively, ${ }^{4}$ suggesting that only patients undergoing complete resection have a chance of long-term survival. Local regional invasion of nearby organs by thymic carcinoma is very common, but vascular invasion (innominate vessel, SVC, pulmonary vessels, or aorta) seems to be the only statistically significant predictor of poor survival according to univariate analysis. ${ }^{2}$ This observation has been confirmed more recently by Tseng and coworkers. ${ }^{3}$ Also, histologic type seems to have an impact on prognosis: well-differentiated thymic carcinoma, defined as a low-grade malignancy, was reported as the most reliable predictor of prognosis. ${ }^{4}$

Resection of thymic carcinoma presenting with intravascular growth in the SVC and right atrium has been rarely reported in the literature ${ }^{5}$ inasmuch as the most common cause of SVC syndrome is extrinsic compression rather than intracaval or intracardiac growth. However, invasion of the SVC and right atrium do not preclude surgery in selected cases because the only chance for long-term survival is complete resection. Surgery should not be indicated if extensive local invasion or distant metastases preclude radical resection. Even for recurrent thymic carcinoma, surgery remains the therapy of choice inasmuch as most of the cases recur even after complete resection.

\section{References}

1. Chung DA. Thymic carcinoma-analysis of nineteen clinicopathological studies. Thorac Cardiovasc Surg. 2000;48:114-9.

2. Blumberg D, Burt ME, Bains MS, Downey RJ, Martini N, Rusch V, et al. Thymic carcinoma: current staging does not predict prognosis. J Thorac Cardiovasc Surg. 1998;115:303-8.

3. Tseng YL, Wang ST, Wu MH, Lin MY, Lai WW, Cheng FF. Thymic carcinoma: involvement of great vessels indicates poor prognosis. Ann Thorac Surg. 2003;76:1041-5.

4. Kondo K, Monden Y. Therapy for thymic epithelial tumors: a clinical study of 1320 patients from Japan. Ann Thorac Surg. 2003;76:878-85.

5. Greene MA, Malias MA. Aggressive multimodality treatment of invasive thymic carcinoma. J Thorac Cardiovasc Surg. 2003;125:434-6. 